\title{
Predicting the Fiber diameter of Spunbonding Nonwovens Via Empirical Statistical methods and Neural Network Model
}

\author{
Bo Zhao \\ College of Textiles, Zhongyuan University of Technology, Henan, Zhengzhou, 450007, China \\ zhaobohenan@sina.com
}

\begin{abstract}
In this paper, the empirical statistical and artificial neural network methods are established. We present a comparative study of two modeling methodological for predicting the fiber diameter of spunbonding nonwovens from the process parameters. The radial basis neural network, which has good approximation capability and fast convergence rate, is employed in this work, and it can provide quantitative predictions of fiber diameter. The effects of process parameters on fiber diameter are also determined by the ANN model. The results show the artificial neural network model yield more accurate and stable predictions than the statistical method, which reveals that artificial neural network technique is really an effective and viable modeling method.
\end{abstract}

\section{Keywords}

Artificial neural network model, statistical model, spunbonding nonwoven, fiber diameter, process parameter.

\section{Council for Innovative Research}

Peer Review Research Publishing System

Journal: INTERNATIONAL JOURNAL OF COMPUTERS \& TECHNOLOGY

Vol. 14, No. 1

www.ijctonline.com, editorijctonline@gmail.com 


\section{INTRODUCTION}

Earlier researchers have been studying the air drawing models of polymer spunbonding process [1-2], and trying to predict the quality of spunbonding nonwovens. However, the reported air drawing models mentioned above was quite elementary, all these models mentioned above were based on using analytical and numerical methods. In fact, there are three modeling methods for predicting spunbonding nonwovens properties: physical, statistical, and artificial neural network model. As a nonlinear problem, predicting the filament fiber diameter of spunbonding nonwovens from the process parameters can be realized by an alternative modeling method, i.e., by using empirical model which includes the statistical, artificial neural network(ANN) model, grey model and etc. Due to its excellent abilities of nonlinear mapping and self-adaptation, ANN provide an alternative to conventional methods. After training by using pass data records from system, a suitably trained ANN has ability to generalize all data. Over the past decades, researchers[3-9] have established ANN model, and used to modeling various textile nonlinear problems. However, the applications of ANN for predicting the filament fiber diameter of spunbonding nonwovens are very scanty.

In this paper, we used two methods, i.e., a statistical model, and an artificial neural network model, to predict the fiber diameter of spunbonding nonwovens with process parameters. Moreover, we analyze and further study the relationships between the process parameters and fiber diameter of spunbonding nonwovens.

\section{THE STATISTICAL MODEL OF SPUNBONDING POLYMER}

As far as statistical model is concerned, for the purpose of constructing a model, the input variables are the polymer throughput rate $(A)$, primary air temperature(B), air suction speed(C), polymer melt temperature(D), Quench pressure(E), venturi $\operatorname{gap}(F)$, and primary cool air $\operatorname{speed}(G)$, All the experimental data are shown in Tab.1. The average of all the ten statistical results is compared with that from the ANN model. The error is taken as the absolute value of the difference between the predicted and measured fiber diameter, expressed as a percentage of the measured fiber diameter. In this work, we established a statistical model for predicting the fiber diameter with a simple linear regression equation of the form

$$
D=a+b_{1} x_{1}+b_{2} x_{2}+b_{3} x_{3}+\ldots \ldots+b_{n} x_{n}
$$

was fitted to the data, the parameters $a$ and $b_{1}, b_{2} \ldots d_{n}$ were constants. The best fitting equation for predicting the fiber diameter of spunbonding nonwovens is found to be

$$
D=4.2018+0.1501 A+0.1961 B+0.1401 C-0.1754 D+0.1987 E+0.1604 F-0.1367 G
$$

\section{THE ANN MODEL OF SPUNBONDING POLYMER}

\section{Neural Network Model}

The multiple perception is a network model in which the neurons are configured in layers, whereby the neurons of a layer are generally all connected with the neurons of the next layer. Because connections exist only from the input layer in the direction of the output layer, this is a feed-forward network. This network is able to process analogue input patterns and to learn in a supervised mode, employing the back-propagation algorithm. For this reason, it is also frequently referred to as a back-propagation network. The multiple perception is kind of neural network that allows representation of the relations between input and output values. The neural network approximates every nonlinear mapping of the form $y=f(x)$. It has been proven that the multiple perception with the back-propagation learning rule is a universal function approximator[10-11]. Figure 1 shows the structure of a two -layer ANN.

\section{Multiple Perception Topology and Levenberg-Marquardt Algorithm}

The multiple perception (MLP) cascading in Figure 1 is developed to predict the fiber diameter. The input vector is 
represented by $p$. The output of the first layer (hidden layer) $a^{1}$ is the input to the second layer (output layer), and $a^{2}$ is the output of the net. The weight matrix, bias vector, and transfer function for the first layer are written as $W^{1}, b^{1}$, and $f^{1}$, while $W^{2}, b^{2}$, and $f^{2}$ are for the second layer. $R$ is the number of input neurons, and $S^{M}$ represents the number of neurons of the Mth layer. Each layer may have a different number of neurons and even different transfer functions. The performance index of the multiple perception is the mean square error.

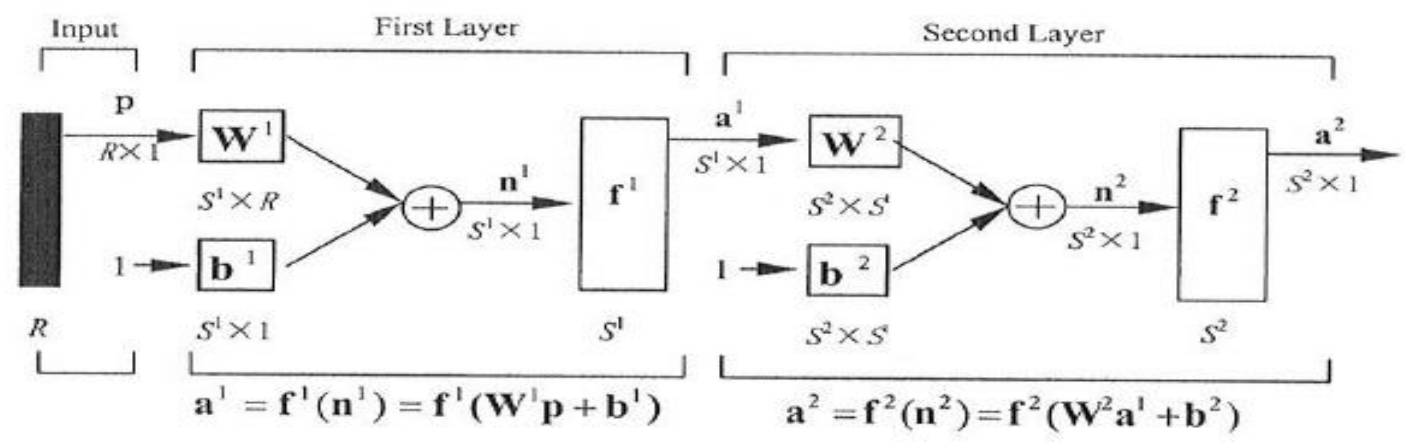

Fig 1: Topology of two-layer perception

In most cases, the standard back-propagation algorithm is used to minimize the mean square error. The mean square error back-propagation is a kind of approximate steepest descent algorithm, so it converges very slowly. In this work, in order to accelerate the convergence, we use the Levenberg-Marquardt algorithm based on the Gauss-Newton method to train the neural network. Due to the second derivation being used, convergence is accelerated.

The performance index of multiple perception is defined as the following forms:

$$
F(x)=\sum_{q=1}^{Q}\left(t_{q}-a_{q}\right)^{T}\left(t_{q}-a_{q}\right)=\sum_{q=1}^{Q} e_{q}^{T} e_{q}=\sum_{q=1}^{Q} \sum_{j=1}^{S^{M}}\left(e_{j, q}\right)^{2}=\sum_{i=1}^{N}\left(v_{i}\right)^{2}=v^{T}(x) v(x)
$$

Where $t_{q}$ is the target of the MLP, $a_{q}$ is the output of the MLP, $e_{j, q}$ is the $j$ th element of the error for the $q$ th input-output pair, $v$ is the error vector, and

$$
\begin{gathered}
N=Q \times S^{M} \\
v^{T}=\left[v_{1} v_{2} v_{3} \ldots v_{N}\right]=\left[e_{1,1} e_{2,1} \ldots e_{S^{M}, 1} e_{1,2} . . e_{S^{M}, Q}\right] \\
x^{T}=\left[x_{1} x_{2} \ldots x_{n}\right]=\left[w_{1,1}{ }^{1} w_{2,1}{ }^{1} \ldots w_{S^{1}, R}{ }^{1} b_{1}{ }^{1} \ldots b_{S^{1}}{ }^{1} \ldots w_{1,1}{ }^{M} \ldots w_{S^{M}, R}{ }^{M} b_{1}{ }^{M} \ldots b_{S^{M}}{ }^{M}\right] \\
n=S^{1}(R+1)+S^{2}\left(S^{1}+1\right)+\ldots+S^{M}\left(S^{M-1}+1\right)
\end{gathered}
$$


According to the Levenberg-Marquardt algorithm, $x$ is calculated by the fellowing correlation:

$$
x_{k+1}=x_{k}-\left(\nabla^{2} F(x)\right)^{-1} \times \nabla F(x)=x_{k}-\left[J^{T}\left(x_{k}\right) J\left(x_{k}\right)+u_{k} I\right]^{-1} J^{T}\left(x_{k}\right) v\left(x_{k}\right)
$$

Where $J(X)$ is the Jacobian matrix, $I$ is the unit matrix, and is the constant.

\section{Experimental}

The polymer used in the experimental runs is YS-835 polypropylene (PP) pellet with a melt index (MI) of $35 \mathrm{~g} / \mathrm{min}$. The mean value of the fiber diameters was considered the final fiber diameter of a polypropylene (PP) nonwoven sample. All the samples tested were conditioned for 24 hours at $65 \% \mathrm{RH}$ and $20 \pm 5{ }^{\circ} \mathrm{C}$ before evaluation.

\section{RESULTS AND DISCUSSION}

\section{Predicting the fiber diameter}

The learning ability of a neural network is a complicated issue. It depends on the structure connections, the transfer functions, the number of hidden layers, and the number of hidden neurons in every hidden layer. By trial and error, we built the 7-2-1(seven input neurons, two hidden neurons, and one output neurons) MLP, where the input neurons consisted of the polymer throughput rate(A), primary air temperature(B), air suction speed(C), polymer melt temperature(D), Quench pressure $(E)$, venturi gap $(F)$, and primary cool air $\operatorname{speed}(G)$, and the the fiber diameter constituted the output neuron. The learning rate was set to 0.01 . All values were scaled between -1 and 1 beforehand. The transfer function for the first layer was $\Psi(x)=\left(e^{x}+e^{-x}\right) /\left(e^{x}+e^{-x}\right)$ and $\Phi(x)=x$ for the second layer. The program was written in Matlab 7.0.

Out of sixty samples, forty-five were chosen as the training set at random, while the rest were the testing set. The testing samples were not used to trained the model. Table 1 shows the predicted and measured outputs, and errors for testing samples.

Table 1. Comparison of measured and predicted diameter of various models for the testing sets

\begin{tabular}{|c|c|c|c|c|c|}
\hline \multirow[t]{2}{*}{ No. } & \multirow{2}{*}{$\begin{array}{c}\text { Measured } \\
\text { diameter } \\
\text { (um) }\end{array}$} & \multicolumn{2}{|c|}{ ANN model } & \multicolumn{2}{|c|}{ Statistical model } \\
\hline & & $\begin{array}{c}\text { Predicted } \\
\text { diameter } \\
\text { (um) }\end{array}$ & $\begin{array}{c}\text { Error } \\
(\%)\end{array}$ & $\begin{array}{l}\text { Predicted } \\
\text { diameter } \\
\text { (um) }\end{array}$ & $\begin{array}{c}\text { Error } \\
(\%)\end{array}$ \\
\hline 1 & 22.826 & 22.819 & -0.031 & 22.932 & 0.464 \\
\hline 2 & 21.383 & 21.380 & -0.014 & 21.403 & 0.094 \\
\hline 3 & 23.038 & 23.044 & 0.026 & 23.157 & 0.517 \\
\hline 4 & 23.282 & 23.285 & 0.013 & 23.082 & -0.859 \\
\hline 5 & 23.621 & 23.624 & 0.013 & 23.704 & 0.351 \\
\hline 6 & 22.637 & 22.642 & 0.022 & 22.559 & -0.345 \\
\hline 7 & 22.450 & 22.444 & -0.027 & 22.350 & -0.445 \\
\hline 8 & 23.262 & 23.259 & -0.013 & 23.457 & 0.838 \\
\hline
\end{tabular}




\begin{tabular}{|c|c|c|c|c|c|}
\hline 9 & 23.342 & 23.335 & -0.030 & 23.482 & 0.599 \\
10 & 22.937 & 22.939 & 0.009 & 22.757 & -0.752 \\
\hline $\begin{array}{c}\text { Maximum of error } \\
\text { absolute value }\end{array}$ & & & 0.031 & & 0.859 \\
\hline $\begin{array}{c}\text { Average(absolute } \\
\text { value) }\end{array}$ & & & 0.0032 & & 0.0462 \\
\hline $\begin{array}{c}\text { Average of error } \\
\text { absolute value }\end{array}$ & & & 0.0198 & & 0.5264 \\
\hline
\end{tabular}

\section{Analyzing Prediction Result between two Modeling Methods}

Table 2 gives the measured fiber diameter, predicted fiber diameter and prediction error of two models for the testing sets .It can be seen from this table that the performance of the ANN model performs better than the statistical model. At the same time, it also can be seen from this table that the average error of the ANN model (giving average error of $0.0032 \%$ ) is much lower than that in the statistical model $(0.0462 \%)$. It is worthwhile to note that the maximum of error absolute value and the average of error absolute value are much larger in the statistical model $(0.859 \%$ and $0.5264 \%$, respectively) than those in the ANN model $(0.031 \%$ and $0.0198 \%$, respectively). In all ten cases, the absolute value of error in the ANN model is the lower in the two models, which means that the prediction errors of the statistical model are more discrete than those of the ANN model.

As far as the maximum of error absolute value and average error and average of error absolute value are concerned, it is obvious that the ANN model performs better than statistical model. Some reasons may be as follows. (1) The statistical model derives regression equations by fitting to historical data gathered from experiments. The statistical model is very easy to use, but it should not be used for predicting fiber diameter outside the range of the data used to develop the model. Furthermore, it is expensive and time consuming to conduct experiments and gather data. (2) The ANN model have excellent abilities of nonlinear mapping and self-adaptation, ANN can provide an alternative to conventional methods. After training by using pass data records from system, a suitably trained ANN has ability to generalize all data. Thus, it can solve control processes that are hard to deal with by mathematical models. Furthermore, when new data belonging to additional levels of input/output pairs are available, the ANN model can be updated by retraining it with both previous and new data.

\section{CONCLUSIONS}

We have analyzed modeling methods for predicting the fiber diameter of spunbonding nonwovens. We use two models, i.e., statistical model and artificial neural network model, to predict fiber diameter. The statistical model is easier to be established and understood than the ANN model, it may be used for intuitive or quantitative analysis. The ANN model predicts fiber diameter using the following parameters as inputs: the polymer throughput rate, quench pressure and air suction speed etc. The results show that the predicted and experimental values agree well, the ANN model produces more accurate and stable predictions than the statistical models, which also reveals that the ANN model technique is really an effective, an excellent and a viable modeling method for predictors. At the same time, the experimental results also demonstrate the great potential of this research for the computer-assisted design of spunbonding technology.

\section{REFERENCES}

[1] Hajji, N. and Spruiell, J. E.1992. Modeling of the reicofil spunbonding process. INDA J. Nonwovens Res. 4 (January 1992), 16-21.

[2] Misra S. and J. E. Spruiell, J. E. 1993. Investigation of the spunbonding process via mathematical modeling. INDA J. 
Nonwovens Res. 5(September 1993), 13-19.

[3] Debnath S., Madhusoothanan M. and Srinivasamoorthy V. R. 2000. Prediction of air permeability of needle-punched nonwoven fabrics using artificial neural network and empirical models. Ind. J. Fiber Textile Res. 25(September 2000), 251-255.

[4] Ertugrul, S. 2000. Predicting bursting strength of cotton plain knitted fabrics using intelligent technique. Textile Res. J. 70(July 2000), 845-851.

[5] Guba, A. 2001. Predicting yarn tenacity: A comparison of mechanistic, statistical and neural network models. J. Text. Inst. 92(March 2001), 139-145.

[6] Ramesh M. C. 1995. The prediction of yarn tensile properties by using artifical neural networks. J. Textile Inst. 98(January 1995), 459-469.

[7] Zeng, Y. C. 2004. Predicting the tensile properties of air-jet spun yarns. Textile Res. J. 74(August 2004), 689-694.

[8] Majumadr P. K.2004. Predicting the breaking elongation of ring spun cotton yarns using mathematical, statistical, and artificial neural network model. Textile Res. J. 74(August 2004), 652-655.

[9] Chen T., Wang X. B. and Huang X. B. Applying artificial neural network model on studying the filtration properties pf melt blown nonwovens: comparison with statistical method, Proc. of The Textile Institute 84th World Conference, 2005, pp. 1006-1013.

[10] D. E. Rumelhat: Eds., Parallel distributed processing-explorations in the microstructure of cognition, vol. 1, Foundations, MIT Press, Cambridge, MA, 1986, p.563-670.

[11] P. D. Wasserman: Advanced methods in neural computing, Van Nostrand Reinhold, NY, 1993, p.267-370.

\section{Acknowledgement}

The author expresses his sincere gratitude to the processor Shi Feng-jun of Zhongyuan University of Technology for his unwearied and assistance during the researching and studying. At the same time the author also appreciates all other assisters during the whole course.

\section{Corresponding author: Dr. Bo Zhao}

Mailing Addresss: College of Textiles, Zhongyuan University of Technology, No.41 Zhongyuan Road, Zhengzhou, Henan 450007, People's Republic of Chin

E-mail: zhaobohenan@sina.com or zhaobohenan@163.com 